\title{
An original Approach to Retrofitting A Post and Core Restoration to an Existing bridge
}

\author{
Debbabi Imen $^{1^{*}}$, Saafi Jilani ${ }^{2}$, Nouira Zohra ${ }^{2}$, Harzallah Belhassen ${ }^{2}$ and Cherif Mounir ${ }^{2}$ \\ ${ }^{1}$ Resident, Department of Fixed Prosthodontics, Dental Clinic of Monastir, Tunisia \\ ${ }^{2}$ Professor, Department of Fixed Prosthodontics, Dental Clinic of Monastir, Tunisia
}

*Corresponding author: Debbabi Imen, Department of Fixed Prosthodontics, Dental Clinic of Monastir, Tunisia, Tel: +21627430478, E-mail: Debbabiimen@yahoo.fr

Citation: Debbabi Imen, Saafi Jilani, Nouira Zohra, Harzallah Belhassen, Cherif Mounir (2018) An original Approach to Retrofitting- A Post and Core Restoration to an Existing bridge. J Oral Health Dent Sci 2: 202

Article history: Received: 19 February 2018, Accepted: 11 April 2018, Published: 13 April 2018

\begin{abstract}
After many years of cementation of a conventional bridge, it is possible that patients may present with fractured abutments teeth due to many reasons including recurrent caries, trauma and debonding composite. In this study an original technique for retrofitting an old bridge using duralay resin as models to perform two casted posts and cores by a direct technique with which the additional time and cost of preparing a new bridge can be avoided.
\end{abstract}

Keywords: Abutment; Fracture; Retrofitting; Duralay resin

\section{Introduction}

One problem that may arise in full-coverage restorations is clinical crown fracture. Fracture of an endodontically treated and crowned tooth in the esthetic zone may be embarrassing to both the patient and clinician [1]. Fixed partial denture abutments severely damaged by dental caries or fracture present a challenge to the dentist [2]. The fixed partial denture may need to be removed for endodontic treatment with post and core fabrication [2]. Several methods have been described and introduced in literature to reproduce the original contour of the tooth for retrofitting an existing crown [3-6].Thus, if a completely sound margin and at least $2 \mathrm{~mm}$ of coronal structure remain (ferrule), a fractured tooth may possibly be retreated by a repair. The existing crown should fit precisely on the pre-existing finish line. Therefore, when one of the abutments in a fixed partial denture (FPD) or splinted crowns fractures, then the repaired core should be placed exactly in the same location as the previous core [4]. Post and core followed by crown is a common treatment option to restore the form, function, and esthetics of an endodontically treated tooth [5]. Retrofitting the post and core greatly improves the stabilization of the prosthesis [2].

\section{Case report}

A 46-year healthy female presented to the department of fixed prosthodontics at the dental clinic of Monastir in emergency to recement her veneered bridge. The bridge has 3 retainers on canine \#13, second premolar \#14 and first molar \#16 and a pontic replacing the first premolar \#14. It was loosened partially on the side of the canine. It was very mobile but in good condition. According to the patient, the bridge dates from 6 years. Moreover, the patient seeked for a quick and urgent aesthetic and functional solution. the patient brought with her an old panoramic X-ray (Figure 1). The bridge was very mobile that's why it was easily and completely removed by the Manual Crown and Bridge Remover which was fixed in the palatal side to avoid the detachment or the fracture of the veneering buccal surfaces. The bridge does not present any cracks after the use of crown remover (Figure 2).

Clinical examination of the abutments teeth showed that the canine was dilapidated. Its decay is supragingival. At the level of the tooth persists a screw post which has been unscrewed using a Classic smooth flat pliers. The \#15 remained as a root tip with softened walls and a very deep caries. Its prognosis is unfavorable so we decided to extract it. The \#16 exhibited extensive restoration with composite resin (Figure 3). Occlusion exam revealed a stable maximum intercuspation. 


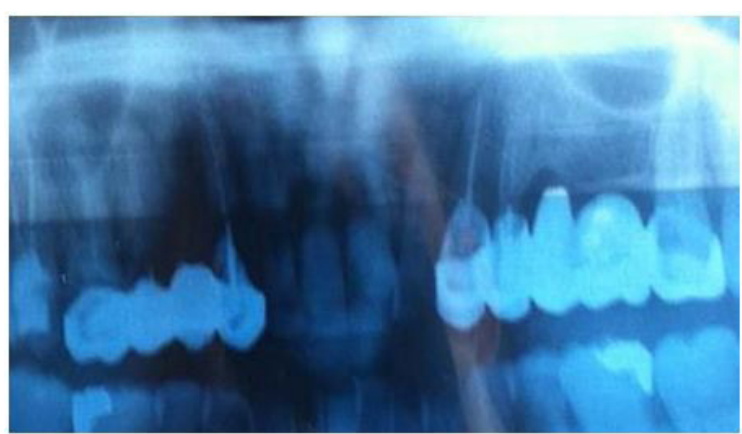

Figure 1: Panoramic radiography

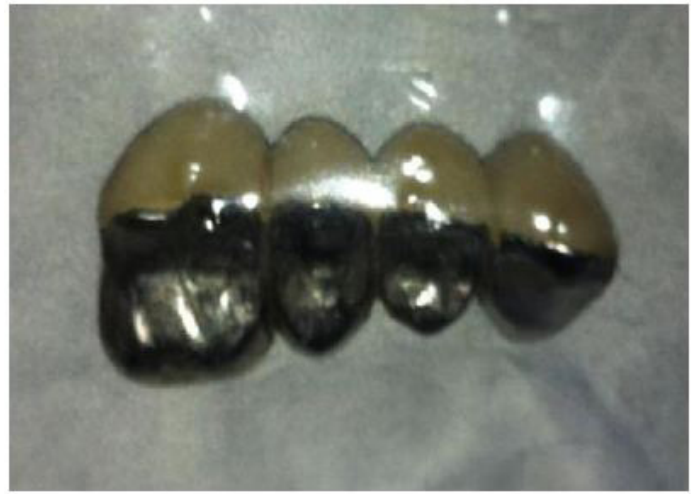

Figure 2: Bridge was removed

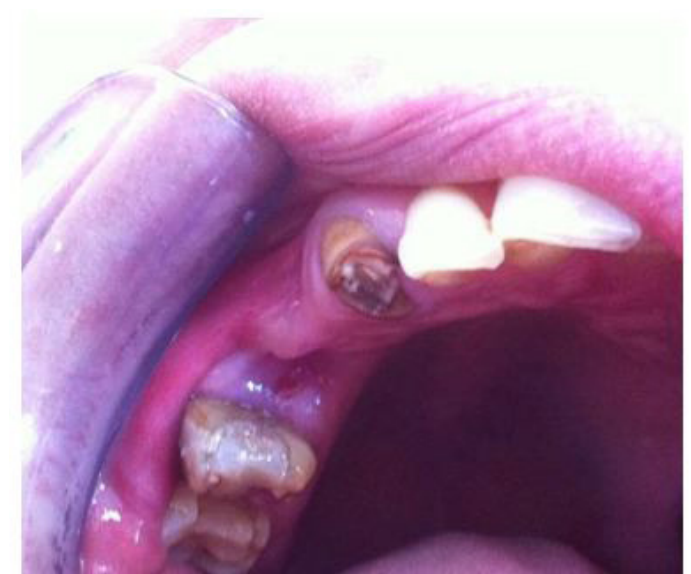

Figure 3: Clinical examination of the abutments after decementation of the old bridge and the extraction of the 15

Radiographic evaluation demonstrated an excellent bone support for the remaining teeth \#13 and \#16 [7]. The canine and molar are properly treated endodontically with acceptable treatment and sufficient remaining coronal tooth structure. The Eatch abutment tooth was thouroghly investigated and residual caries, and existing restoration and cement were removed [8].The finish lines of the two teeth (\#14 and \#16) were preserved after peripheral preparation (Figure 4).

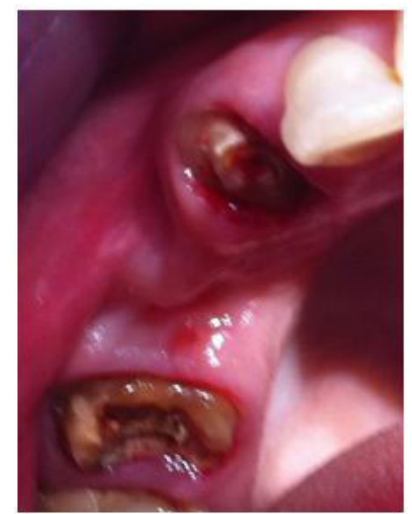

Figure 4: The finish lines of the two teeth (\#14 and \#16) are preserved after peripheral preparation 
In this case, the pontic is above the gum - which makes it very easy to clean using interdental brush. The problem in this option is that there is a very noticeable gap between gum and pontic, which is not pleasant to look at (Figure 5). Thankfully, the gap is not visible when smiling so it does not cause an aesthetic problem for the patient to whom the situation has been explained (Figure 6). The patient asked to use the old bridge as a quick and temporary solution.

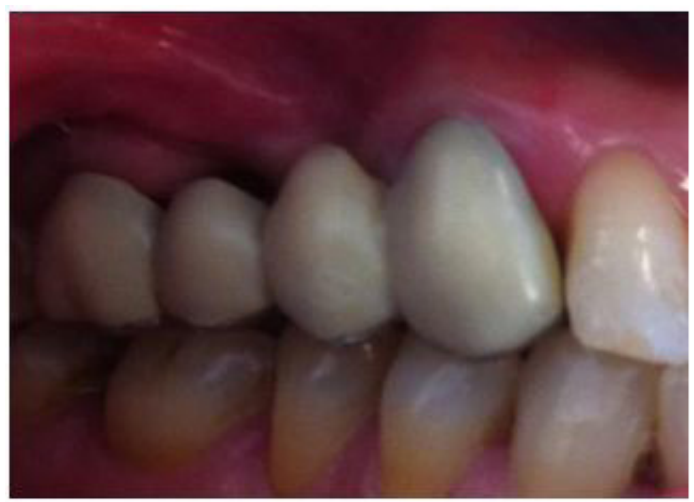

Figure 5: Noticeable gap between gum and pontic

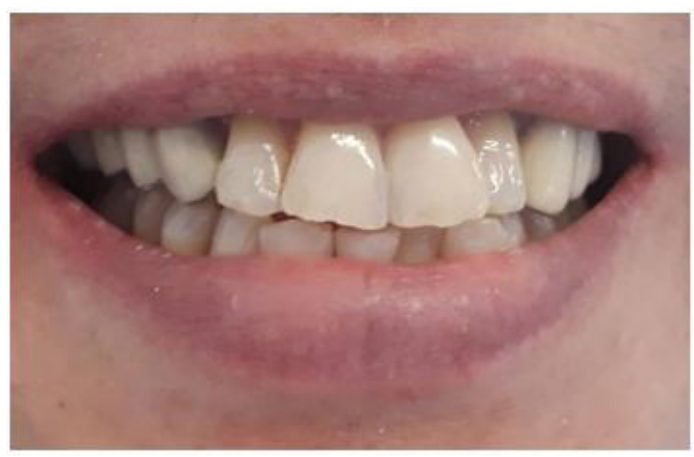

Figure 6: The gap is not visible when smiling

The procedure is to retrofit the inner surfaces of the retainers of the same bridge by duralay resin in maximum intercuspation. The final decision was made with the full cooperation and informed consent of the patient.

1st day:

The patient was sent to the Department of Medicine and Oral Surgery to extract the \#15. An analgesic and a mouthwash have been prescribed to improve the state of the gum and relieve postoperative pain.

Frequently, the existing of fixed partial denture can be recycled by acryl resin using or no metal posts converting the original fixed partial denture to an effective provisional restoration [2].

\section{After 4 weeks:}

The healing of the gum related to the socket was well improved. After selecting an appropriate size of plastic posts (Para-Post, Whaledent International, New York, N.Y.), they were inserted into the prepared root canals. The plastic posts were rebazed by Coating the post with an autopolymerizing acrylic resin material (Duralay, Reliance Dental Mfg Co, Alsip, Ill.) to ensure better adaptation to the teeth structure (post in post space of \#13 and the palatal post space of the \#16) (Figure 7). The length of the post were adjusted by cutting them with a disk so they do not interfere with the inner surfaces of the retainers which can jeopardize accurate seating and appropriate occlusion (Figure 8).

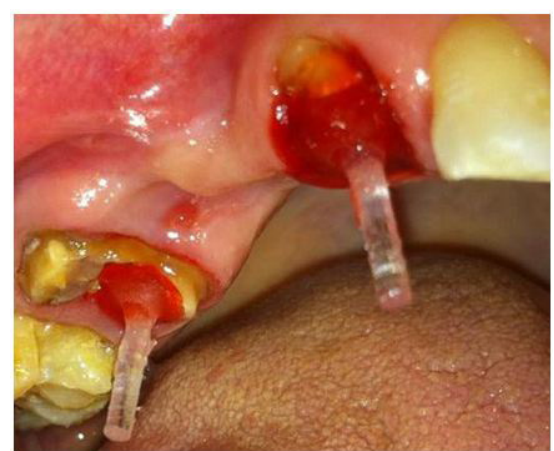

Figure 7: The plastic posts were rebased with duralay resin 


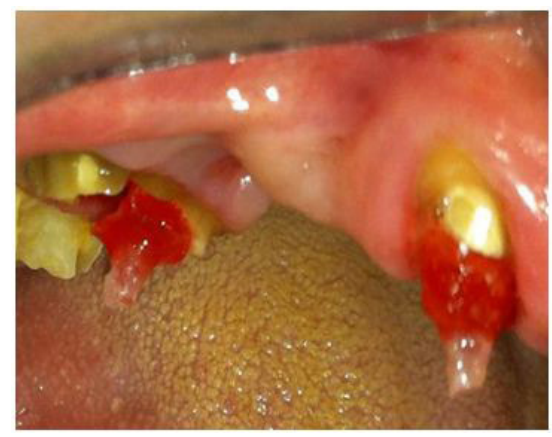

Figure 8: The posts are cut

A layer of lubricant was applied in each inner surface of retainers and to the teeth structures to facilitate the desinsertion of the bridge after the setting time of the resin. The bridge is tried in maximum intercuspation (MI) (Figure 9).

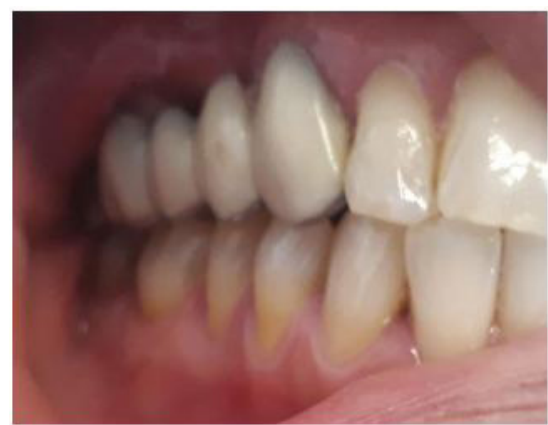

Figure 9: The bridge is tried in maximum intercuspation

The duralay resin material was then mixed and filled in the inner surfaces of the retainers, then placed over the plastic posts and the patient was asked to close in the position of maximum intercuspation (MI) to avoid sur-occlusion until the resin material completely polymerizes then removed (Figure 10).

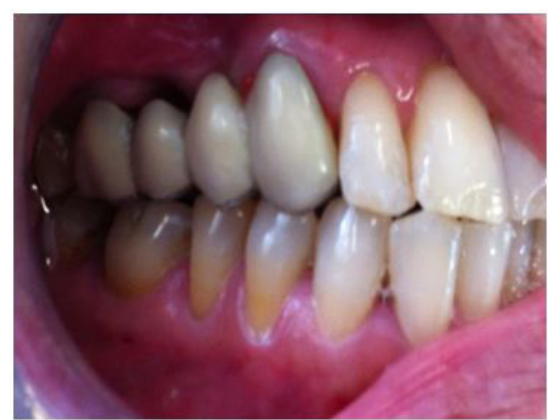

Figure 10: The patient was asked to close in the position of maximum intercuspation (MI)

The refabricated Duralay posts and cores were checked so that there was no deficiency of the material and that the contour of the previous cast post and core was reproduced and excessive core material may remain over the margin was removed using a probe. The setting time of the Duralay resin is $5 \mathrm{~min}$. the models of 2 posts and cores were refined using dental diamond bur on turbine under irrigation to create additional cement space between the abutments and the retainers, then sent to the laboratory to be casted in metal (Figure 11,12 and 13) [5]. Obviously at this step adding grooves or boxes to the refabricated duralay post and core (new abutments) limits the paths of placement, so retention is increased [9].

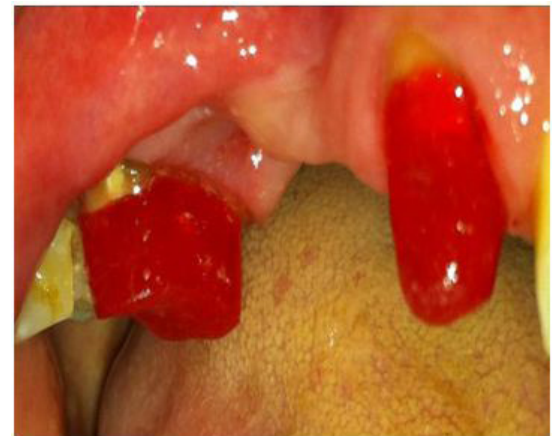

Figure 11: Models of 2 casted post and core after refining 


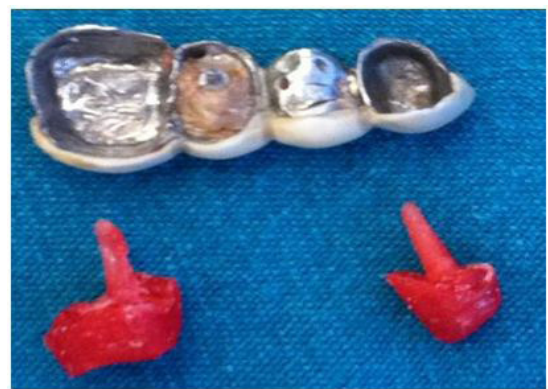

Figure 12: Old bridge with two models of two duralay posts and cores
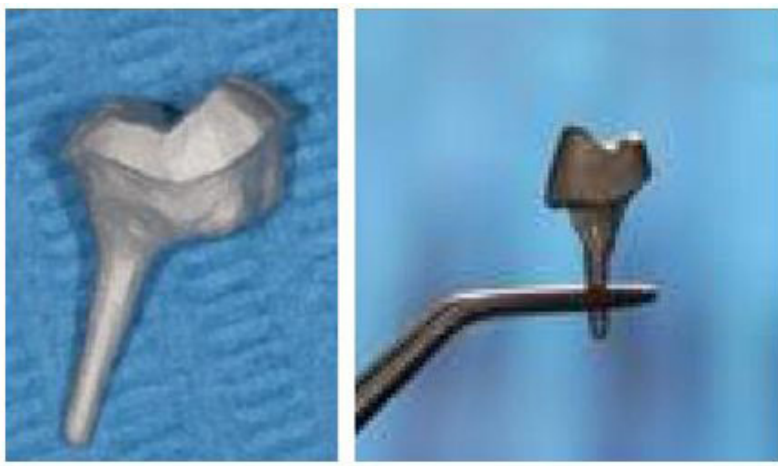

Figure 13: The models of 2 posts and cores casted in metal

In the lab, the fitting surface of retainer on \# 15, already extracted, was cleaned and most effectively prepared by air-abrading with $50 \mathrm{~mm}$ of alumina and in the clinic it was filled with a light cure composite resin to be transformed into Pontic (Figure 14).

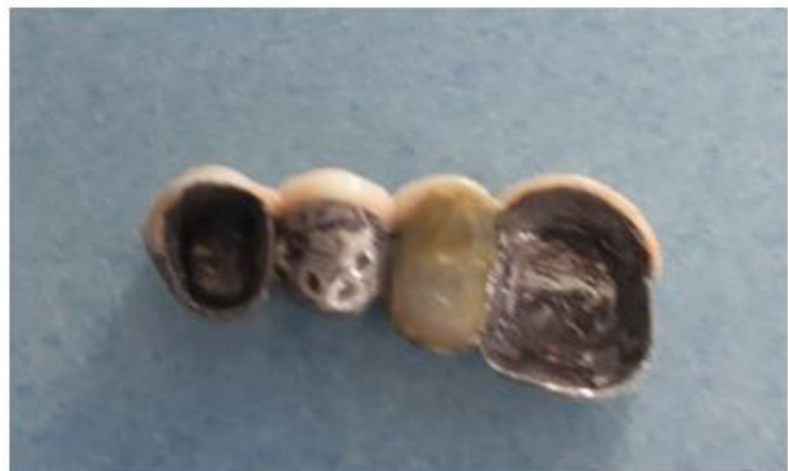

Figure 14: Light cure composite resin filled in the inner surface of tooth \#15

\section{The next day:}

The casted posts and cores were tried in the mouth without the bridge to ensure their perfect adaptation, then in a second time with the bridge in situ to assess the absence of over-occlusion. Finally, they were cemented with adhesive glass ionomere cement (Figure 15). In the last time, the bridge is cemented using zinc phosphate cement (Figure 16).

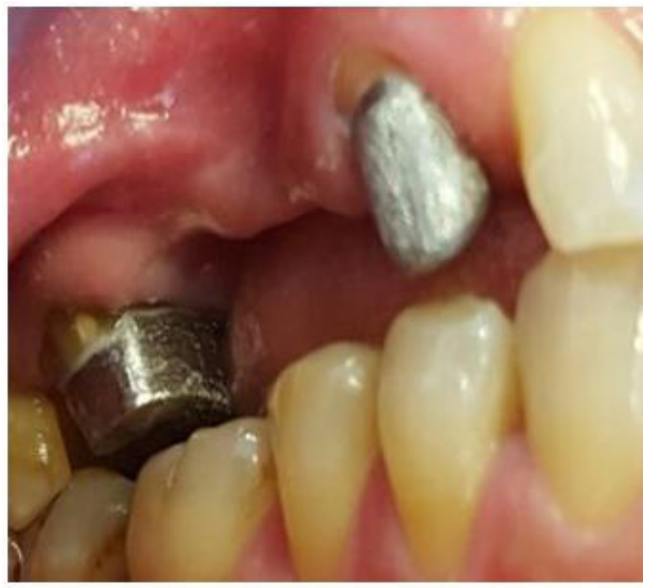

Figure 15: The 2 casted post and core are cemented 


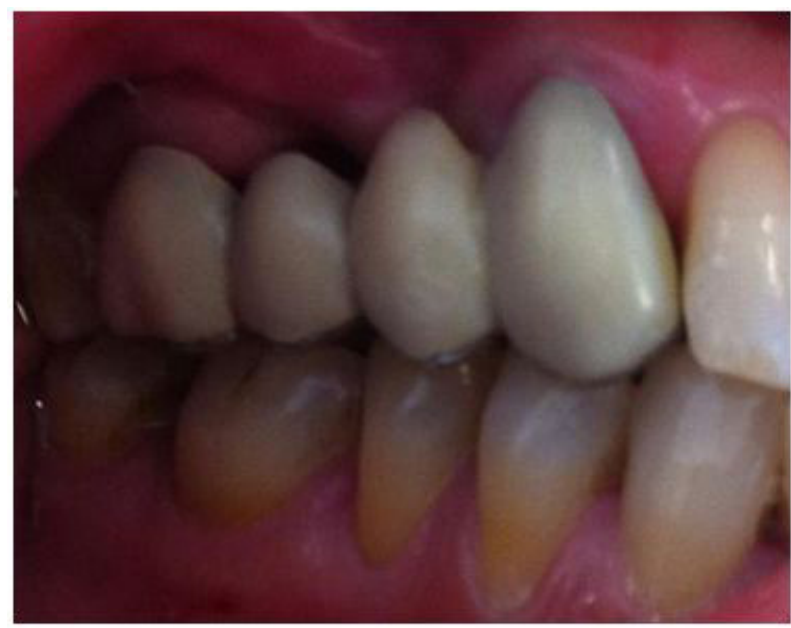

Figure 16: The bridge was cemented

\section{Discussion}

Failure occurred on endodontically treated teeth are due to decreased or altered tooth structure caused by caries and/or previous restorations, fracture or trauma and decreased moisture [10]. Remaining of an unaltered finition line (margin) on the two abutments is paramount to use this old bridge for reconstruction of two casted posts and cores.

In this clinic case and according to Ant's law the replacement of two premolars using two abutments canine and molar and with no bone loss was a suitable solution [11].

The abutments permit to withstand normal axial forces as well as oblique ones during function.

Different approaches of various authors have been suggested, in literature, to retrofit the crown: Sabbak used a clear polyvinyl siloxane (PVS) impression material as a matrix [5]. Chan adapted a polytetrafluoroethylene sheet as a separating medium onto the inner aspect of the crown to leave additional cement space between the crown and the core [4]. Berksun used manually fabricated plastic foil as a template for rebuilding the core, Jahangiri and Feng used the original die or its replica and a vinyl polysiloxane matrix $[3,6]$. Rosen $\mathrm{H}$, in his article published in 1998, described a chair side procedure for retrofitting posts and cores and, at the same appointment, converting the original fixed partial denture to an effective provisional restoration [1].

This article describes a simple procedure of recording contours of cast posts and cores that need to be refabricated, using duralay resin under occlusal pressure by retrofitting the inner surface of retainers on fractured abutments which can be a preferred option. Acrylic resin materials undergo volumetric contraction (shrinkage) during the polymerization process. One study reported that $80 \%$ of all the shrinkage of Duralay resin occurs before 17 minutes and $95 \%$ before 3 hours at room temperature [12].

In addition, they found high polymerization shrinkage as a result of a high monomer/polymer ratio and suggested to use a mix as thick as possible to minimize the worst effects of polymerization.

In this clinic case, Duralay resin was used in a thick consistency as was recommended and the excessive use of monomer was avoided as much as possible [5].

To leave additional cement space between the abutments and the retainers we have used a rotary diamond bear to trim slightly and empirically the surfaces of the two casted posts and cores.

As an existing prosthesis is reused, the issues of additional time and cost for repreparation, reimpression, and new crown are eliminated [1].

Simple to perform [5]. Retrofitting the post and core greatly improves the stabilization of the interim as well as the remake of the definitive fixed partial denture [2]. Frequently, the existing fixed partial denture can be recycled as a provisional restoration [2].

\section{Conclusion}

This technique is depending on the extent and severity of the fracture, retrofitting the crown due to a fracture of abutment can be a preferred option. This technique which can be termed as $\langle<$ retrograde technique $\rangle>$, simple in appearance, needs more accurency. Nevertheless, it needs relatively reduced chairside time and cost. Frequently, the existing fixed partial denture can be recycled as a provisional restoration [2].

\section{References}

1. Lee JH (2017) A Digital Approach to Retrofitting a Post and Core Restoration to an Existing Crown. J Prosthodont doi: 10.1111/jopr.12720.

2. Rosen H (1998) Dissolution of cement, root caries, fracture, and retrofit of post and cores. J Prosthet Dent 80: 511-3. 
3. Jahangiri L, Feng J (2002) A simple technique for retrofitting a post and core to a crown. J Prosthet Dent 88: 234-5.

4. Chan DC (2003) Technique for repair of multiple abutment teeth under preexisting crowns.J Prosthet Dent 89: 91-2.

5. Sabbak SA (2000) Simplified technique for refabrication of cast posts and cores. J Prosthet Dent 83: 686-7.

6. Berksun S (2005) Rebuilding core foundations for existing crowns using a custom-made template. J Prosthet Dent 93: 201-3.

7. Doan PD, Goldstein GR (2007) The Use of a. Diagnostic Matrix in the Management of the Severely Worn Dentition. J. Prosthodont 16: 277-81.

8. Christensen, G.J (1996) When to use fillers, build-ups or posts and cores. J Am Dent Assoc 127: 1397-8.

9. Galun EA, Goodacre CJ, Dykema RW, Moore BK, Sowinski LL (1986) The contribution of a pinhole to the retention and resistance form of veneer crowns. J Prosthet Dent 56: 292.

10. Dorothy McComb (2008) Restoration of the Endodontically Treated Tooth. Ensuring Continued Trust; Dispatch; February/March, 2018.

11. Ante IH (1926) The fundamental principles of abutments. Mich State Dent Soc Bull 8: 14.

12. Mojon P, Oberholzer JP, Meyer JM, Belser UC (1990) Polymerization shrinkage of index and pattern acrylic resins. J Prosthet Dent 64: 684-8. 